\title{
The Immunologic Role of Vitamins C and D and Their Potential in the Management of COVID-19 Infections
}

\author{
Chrystal D. Antoine-Frank ${ }^{* 1}$, Rimanatou Seyni Boureima ${ }^{2}$, Kaydeonne T. Ellis ${ }^{3}$, Malcolm (Jr) R. Antoine ${ }^{4}$, \\ Merina Shrestha ${ }^{5}$
}

${ }^{1}$ Department of Anatomical Sciences, St. George's University, True Blue, Grand Anse, St. George, Grenada, West Indies

${ }^{2}$ Department of Anaesthesiology, Zhongnan Hospital of Wuhan University, Wuhan, Hubei, China

${ }^{3}$ Torbay Hospital, Torbay and South Devon NHS Foundation Trust, United Kingdom

${ }^{4}$ Department of Microbiology, St. George's University, True Blue, Grand Anse, St. George, Grenada, West Indies

${ }^{5}$ Department of Cardiology, Zhongnan Hospital of Wuhan University, Wuhan, Hubei, China

*Corresponding author: Chrystal D. Antoine-Frank; cantoinef4@ gmail.com

Received 30 August 2021;

Accepted 13 September 2021;

Published 16 September 2021

\begin{abstract}
The Severe Acute Respiratory Syndrome Coronavirus 2 causes COVID-19 infection. Vitamins C and D have been investigated for their effects on COVID-19 including the severity of it's symptoms and complications. These vitamins may provide some benefits against the Severe Acute Respiratory Syndrome Coronavirus 2 as they modulate the immune system in addition to having anti-viral effects. This review will investigate the effects of vitamins C and D on COVID-19 infection to determine their benefits in the management of patients infected by the Severe Acute Respiratory Syndrome Coronavirus 2.
\end{abstract}

Keywords: vitamin C, vitamin D, ascorbic acid, COVID-19, SARS-CoV-2

\section{Introduction}

COVID-19 is caused by the severe acute respiratory syndrome coronavirus 2 (SARS-CoV-2) virus ${ }^{[1]}$. This disease was first reported in China towards the end of 2019. Following a rapid epidemic spread throughout the mainland of China, the World Health Organization (WHO) labelled the disease a pandemic as numerous countries across the globe became affected ${ }^{[2]}$. Worldwide, there are more than 90 million reported cases of COVID-19 ${ }^{[3]}$. The SARS-CoV-2 virus is mainly transmitted from person-to-person via respiratory secretions suggesting that close contact may be required for successful transmission ${ }^{[4]}$. Symptoms of COVID-19 may include: fever, cough or shortness of breath and fatigue ${ }^{[5,6]}$. Gastrointestinal symptoms may also be observed and in some instances, patients may be asymptomatic ${ }^{[7]}$.

To date, the treatment of COVID-19 has proven quite complicated and this has therefore brought to light two vitamins (vitamins C and D) that may serve some benefits to COVID-19 patients. Vitamin $\mathrm{C}$ is an anti-oxidant that exhibits antiinflammatory properties in addition to promoting immunity. It has already been shown that patients infected with the SARS-CoV-2

$\underline{\text { www.ijirms.in }}$ virus usually experience low serum levels of vitamin $C^{[8]}$. Vitamin D exhibits anti-viral effects and evidence suggests that the metabolites of this vitamin have inhibitory effects on the SARS$\mathrm{CoV}-2$ virus ${ }^{[9]}$. Associations between serum levels of vitamins $\mathrm{C}$ and $\mathrm{D}$ and the development of COVID-19 has already been investigated in COVID-19 patients. Therefore, this review will examine the effects of vitamins C and D on COVID-19 infection and deduce whether or not these vitamins may be beneficial in treating COVID-19 patients.

\footnotetext{
Vitamins C and D

Vitamin $\mathrm{C}$ also called ascorbic acid, is a water soluble vitamin ${ }^{[10]}$. It is an essential vitamin that serves many important roles in the body. Claims have been brought forward to implicate this vitamin in supporting cardiovascular health and fighting malignancies ${ }^{[11-}$

${ }^{14]}$. The use of this vitamin helps in improving immunity as it can act as an anti-oxidant thus neutralizing excess free radicals that may potentially cause tissue damage. It also functions in collagen production and in the synthesis of carnitine and catecholamines [15,16]. Vitamin C supports the production of bile acids from cholesterol ${ }^{[17]}$. Animal sources only contain vitamin $\mathrm{C}$ in small
} 
amounts; therefore, this vitamin is mainly obtained from the consumption of plant based foods such as strawberries, tomatoes, peppers and broccoli. Within the oral cavity, vitamin $\mathrm{C}$ is passively absorbed; however, it is actively absorbed from the gastrointestinal tract $^{[18,19]}$.

Vitamin D, a fat soluble vitamin, exists in two forms: ergocalciferol (vitamin D2) and cholecalciferol (vitamin $\mathrm{D}_{3}$ ). It functions in the body to regulate calcium and phosphorus levels in the blood. Evidence suggests that this fat soluble vitamin plays an essential role in preventing the development of osteoporosis in addition to preventing malignancies and autoimmune diseases. It also promotes cardiovascular health ${ }^{[20]}$. In addition, vitamin D promotes hormone secretion, regulates immune system function and supports cell proliferation and differentiation ${ }^{[21]}$. In humans, the skin epidermis serves as an important endogenous source of vitamin D. In the presence of ultraviolet B (UVB) light, previtamin $\mathrm{D}_{3}$ is produced in skin cells through a non-enzymatic reaction. Subsequent reactions in the skin finally convert previtamin $\mathrm{D}_{3}$ to vitamin $\mathrm{D}_{3}$. Vitamin $\mathrm{D}_{3}$ is then carried in the blood and undergoes conversion to 25 -hydroxyvitamin $\mathrm{D}_{3}\left(25 \mathrm{OHD}_{3}\right)$ in the liver after which, $25 \mathrm{OHD}_{3}$ is converted to 1,25 -dihydroxyvitamin $\mathrm{D}$ $\left(1,25(\mathrm{OH})_{2} \mathrm{D}\right)$ by an enzymatic process in the kidneys ${ }^{[22]}$. Apart from being synthesized by the skin, vitamin D can also be obtained from the consumption of foods such as liver, red meat and oily fishes such as sardines and salmons ${ }^{[23]}$.

\section{The Effects of COVID-19 on the Immune System}

When the SARS-CoV-2 virus binds to receptors within the pulmonary system it activates the immune system leading to a sequence of events and the ultimate release of cytokines. Excess cytokines in response to the SARS-CoV-2 virus within the alveolar of the lungs cause a dysregulation of the immune system leading to the development of ARDS. Higher levels of interleukin (IL) 6 are observed in patients affected by COVID-19 ${ }^{[24]}$. Respiratory complications observed in COVID-19 patients may be attributed to the presence of higher levels of this inflammatory mediator, as IL6 is known to be involved in respiratory failure and multiple organ dysfunction and failure. Patients presenting with severe symptoms of COVID-19 also have higher levels of pro-inflammatory markers such as IL-2, IL-7, granulocyte colony-stimulating factor (G-CSF) and tumor necrosis factor $\alpha(\mathrm{TNF}-\alpha)$ and these patients also experience abnormal immune signaling especially of the Human Leukocyte Antigen DR-isotype (HLA-DR allele) and show major decrease in the number of lymphocytes ( $\mathrm{T}$ and $\mathrm{B}$ lymphocytes) and natural killer cells ${ }^{[25-27]}$.

\section{Vitamins $C$ and $D$ and the immune System}

Vitamin C plays an important role in immunity as it functions to regulate many components of the immune system. White blood cells, like neutrophils, actively take up vitamin $\mathrm{C}$ thus accumulating high intracellular levels of this vitamin [28-30]. Vitamin C plays an essential role in the function of neutrophils and it is believed that high levels of ascorbic acid within these immune cells, serve a protective role against cellular damage from free radicals ${ }^{[31]}$. Furthermore, vitamin $\mathrm{C}$ supports the normal migration of neutrophils to sites of injuries and infections through a process called chemotaxis ${ }^{[32]}$. Low levels of vitamin C, as is commonly observed during an infection, can impair the process of neutrophil migration, therefore compromising appropriate immune responses ${ }^{[33]}$. Evidence also suggests that vitamin $\mathrm{C}$ deficiencies potentially reduce the ability of neutrophils to phagocytose microbes and low levels of this vitamin diminish the ability of neutrophils to destroy invading pathogens, as the generation of reactive oxygen species (ROS) is affected ${ }^{[34]}$. Neutrophil apoptosis and clearance are also affected by the presence of low levels of ascorbic acid ${ }^{[35]}$. In addition to enhancing the functions of neutrophils, vitamin $\mathrm{C}$ demonstrates positive effects on the actions of other white blood cell, as it promotes lymphocyte proliferation ${ }^{[36]}$, supports antibody production and prevents the death of lymphocytes ${ }^{[37]}$. These similar effects are also observed in natural killer cells ${ }^{[38]}$.

Vitamin $\mathrm{C}$ also influences humoral and cell mediated immunity; however, it is more evident that it has greater effects on cell mediated responses. A study that investigated 29 patients with Crohn's disease as means of accessing their immune status following oral supplementations with vitamin $\mathrm{C}$, zinc or placebo, concluded that vitamin $\mathrm{C}$ supplementation improved $\mathrm{T}$ - cell function in study subjects while it had no significant effects on humoral immunity ${ }^{[39]}$. Vitamin $\mathrm{C}$ regulates the production of different inflammatory markers as it has already been demonstrated to enhance the production of anti-inflammatory mediators such as IL-10 while suppressing the production of pro-inflammatory mediators such as TNF- $\alpha$, interferon gamma (IFN- $\gamma$ ) and interleukin 1 beta $(\mathrm{IL}-1 \beta)^{[40,35,33]}$.

Similar to vitamin $\mathrm{C}$, vitamin $\mathrm{D}$ also potentially regulates the immune system. It inhibits the differentiation of precusor B cells into plasma cells ${ }^{[41]}$ and prevents the proliferation of B cells. In addition, it decreases the production of IL- 2 and IFN- $\gamma$ by preventing the proliferation of T-cells, particulary T helper (Th)-1 cells ${ }^{[42]}$ but on the contrary, the presence of vitamin $\mathrm{D}$ enhances the activity of $\mathrm{T}$ helper (Th)-2 cells. Furthermore, vitamin D induces $\mathrm{T}$ regulatory cells and influences the maturation of $\mathrm{T}$ cells shifting away from the inflammatory Th17 phenotype. The overall effects of these actions result in a decreased production of proinflammatory cytokines such as IL-17 and IL-21 while promoting the production of anti-inflammatory cytokines like IL-4, IL-5 and IL-10 ${ }^{[43-45]}$. Moreover, vitamin D prevents monocytes from producing inflammatory mediators such as IL,6, IL-8, IL-12, and TNF- $\alpha{ }^{[43]}$. It also influences the differentiation and maturation of dendritic cells thus preventing the development of autoimmune diseases ${ }^{[44]}$.

Both vitamins $\mathrm{C}$ and D have already been investigated for their effects on infections especially infections of the respiratory system. A randomized double-blinded controlled trial designed to examine the association between vitamin $\mathrm{C}$ supplementation and the common cold recruited 439 subjects. The intervention received was $50 \mathrm{mg}$ (low-dose) or $500 \mathrm{mg}$ (high dose) of vitamin $\mathrm{C}$ supplementation daily. This study demonstrated that the number of cases of the common cold observed was higher in the low dose group as compared to the high does group; however, this study showed no major effects of vitamin $\mathrm{C}$ supplementation on cold duration or severity ${ }^{[46]}$. Furthermore, a systematic literature review was conducted to investigate the effects of vitamin $\mathrm{C}$ in preventing or reducing the duration of upper respiratory tract infections in children. This review included eight randomized controlled trials, with a total of 3135 children aged 3 months to 18 years. It demonstrated that the use of vitamin $\mathrm{C}$ potentially reduced the duration of the assessed outcome by 1.6 days (standardized mean differences $=-0.30[-0.53 ;-0.08], \mathrm{p}=$ $0.009, \mathrm{I} 2=70 \%$ ) in study participants ${ }^{[47]}$. Also, another study was done to assess the effects of vitamin $\mathrm{C}$ on respiratory infections in military personnel and in other subjects that were living under similar conditions to those of military recruits. This analysis showed a $45 \%$ to $91 \%$ reduction in the occurrence of the common cold and an $80 \%$ to $100 \%$ decrease in the occurrence of pneumonia in study subjects who were supplemented with vitamin $\mathrm{C}$, thus implying that vitamin $\mathrm{C}$ may have some protective role to play in respiratory infections ${ }^{[48]}$. Additionally, a meta-analysis which 
examined 9 randomized controlled trials to discover the effectiveness of vitamin $\mathrm{C}$ in treating the common cold showed that the use of extra therapeutic doses of vitamin $\mathrm{C}$ at the beginning of a common cold infection helped to reduce the duration of the infection in addition to reducing the severity of presenting symptoms. These observations were demonstrated regardless of routine supplementation. This study therefore concluded that the use of extra therapeutic doses of vitamin $\mathrm{C}$ may prove beneficial in patients affected by the common cold ${ }^{[49]}$.

Low levels of vitamin D affects the immune system and therefore, has been associated with increased risks for developing infections. Different studies have already demonstrated the beneficial effects of vitamin $\mathrm{D}$ in preventing the occurrence and reducing the severity of symptoms observed in patients suffering from respiratory illnesses. A study was carried out in young Finnish men to investigate the possible existence of an association between vitamin $\mathrm{D}$ deficiency and the development of acute respiratory tract infections. This study demonstrated higher incidences of respiratory infections resulting in lost working days in study subjects that exhibited lower levels of serum vitamin D as compared to control subjects ${ }^{[50]}$. Furthermore, a randomized controlled trial which assessed the effects of vitamin D supplementation on the occurrence of seasonal influenza $A$ in school children, concluded that the incidence of this disease during the winter season may be reduced through the use of vitamin $D_{3}$ supplementation. In this study, more cases of influenza A occurred in control subjects (31 of 167) as compared to study subjects who were supplemented with vitamin D (18 of 167) ${ }^{[51]}$. In addition, one multi-center, randomized, open, controlled clinical trial was done to assess vitamin D for its clinical efficacy and safety in preventing influenza A infections in children. In this study, 400 infants were assigned to receive either low-dose or high-dose vitamin D and it was demonstrated that more cases of influenza A were reported in the low-dose group (78 cases) as compare to the high-dose group (43 cases). It was also noted from this study that cases in the highdose vitamin D group recovered more rapidly from existing symptoms and carried a lower viral load as compared to the cases identified in the low-dose vitamin D group. This study therefore concluded that high-dose vitamin D supplementation was effective in preventing seasonal influenza ${ }^{[52]}$. Moreover, another randomized, double-blind controlled clinical trial was designed to evaluate the effects of vitamin D supplementation during the winter season and early spring on the occurrence of influenza and upper respiratory tract infections. This study which recruited 223 patients with irritable bowel syndrome, concluded that supplementation of vitamin D potentially protects against upper respiratory tract infections ${ }^{[53]}$. Also, a randomized, double-blinded controlled clinical trial to evaluate the role of vitamin $\mathrm{D}$ in influenza and upper respiratory tract infections in 1300 healthy children and adolescence, demonstrated that vitamin D supplementation reduced the risk of respiratory tract infections in study subjects; however, vitamin D was unable to reduce the occurrence of influenza infections ${ }^{[54]}$.

\section{Vitamins C and D and COVID-19}

Vitamin $\mathrm{C}$ has been investigated for its potential benefits in patients presenting with COVID-19 infections and evidence has already suggests a beneficial role of this vitamin in COVID-19 patients. A randomized, controlled clinical trial carried out in three different hospitals in the province of Hubei, China, recruited fiftyfour critically ill COVID-19 patients to investigate the effectiveness of high-dose vitamin $\mathrm{C}$ infusion in the treatment of severely ill COVID-19 patients. Patients who were diagnosed with
COVID-19 were assigned to receive either high-dose intravenous vitamin $\mathrm{C}$ or placebo. This study concluded that the use of highdose vitamin $\mathrm{C}$ ( $24 \mathrm{~g}$ per day for 7 days) may prove clinically effective in the treatment of critically ill COVID-19 patients ${ }^{[55]}$. A case report of a 74 year old woman, diagnosed with COVID-19 and presenting with acute respiratory distress syndrome (ARDS) showed a good prognosis for severely ill COVID-19 patients treated with high-dose intravenous vitamin $\mathrm{C}$. This case demonstrated faster recovery time and reduced length of stay in the intensive care unit (ICU) with shorter mechanical ventilation time [56]. A case series which included 17 COVID-19 patients who received intravenous vitamin $\mathrm{C}$ for the management of their symptoms, concluded that this vitamin can potentially be used in the treatment of patients with moderate to severe COVID-19 infections. This study demonstrated meaningful reductions in the levels of inflammatory markers, in addition to potential reductions in the $\mathrm{FiO} 2$ (fraction of inspired oxygen) requirements in patients treated with vitamin $\mathrm{C}^{[57]}$. One meta-analysis which included eight clinical trials and a total of 685 patients presented strong evidence to suggest that the use of vitamin $\mathrm{C}$ decreases the length of time required for mechanical ventilation in severely ill COVID-19 patients ${ }^{[58]}$. To date, further studies are being designed and executed to investigate the potential benefits of using vitamin $\mathrm{C}$ in COVID-19 patients. Published data from these investigations will potentially present better insight on the safety, tolerability and efficacy for the use of vitamin $\mathrm{C}$ in the successful treatment of COVID-19. The accumulated knowledge will provide better guidelines in the care of COVID-19 patients.

Evidence suggests that vitamin D may also have some beneficial effects in the management of COVID-19 infections. These claims may be supported by the fact that the initial outbreak of this disease occurred during the winter season when serum vitamin D levels are usually found to be significantly lower. In addition, it is observed that the number of COVID-19 cases identified in the Southern Hemisphere are much lower towards the end of the summer season. Furthermore, the number of cases of ARDS observed to be associated with COVID-19 infections are much greater in individuals with lower vitamin D levels. Older patients and patients presenting with severe comorbidities are more likely to present poorer prognoses when affected by COVID-19 and it is also known that these patients are more likely to experience vitamin D deficiencies ${ }^{[59]}$.

One study was carried out at an urban academic medical centre to assess the association between vitamin $\mathrm{D}$ status prior to COVID-19 testing and potential COVID-19 test outcomes. In this retrospective cohort study, vitamin D levels were measured one year before COVID-19 testings were done and 489 study participants were categorized based on their vitamin D status as being likely deficient, likely sufficient or uncertain. Results from this study demonstrated higher rates of COVID-19 infections in the deficient group as opposed to the sufficient group $(21.6 \%$ (95\% CI, $14.0 \%-29.2 \%$ ) vs $12.2 \%(95 \% \mathrm{CI}, 8.9 \%-15.4 \%)$. Therefore, it was concluded that the risk for COVID-19 increased in the presence of vitamin D deficiency ${ }^{[60]}$. Another retrospective, observational study, which examined the medical records of 42 study subjects with acute respiratory failure secondary to COVID-19 infection, treated in the Respiratory Intermediate Care Unit (RICU) of the Policlinic of Bari from March, 11 to April 30, 2020, showed that higher rates of hypovitaminosis D were observed in COVID-19 patients suffering from ARDS and that mortality rates were higher in patients who presented with severe vitamin D deficiency ${ }^{[61]}$. A parallel pilot randomized open label, double-masked clinical trial carried out at Reina Sofia University Hospital, C'ordoba Spain 
enrolled 76 consecutive patients hospitalized with COVID-19 infection. This study assessed the effects of calcifediol treatment on ICU admission and mortality rate among Spanish patients hospitalized for COVID-19. Results from this evaluation demonstrated that the use of high doses of calcifediol or 25hydroxyvitamin $\mathrm{D}$, decreased the rate for ICU admissions in hospitalized COVID-19 patients ${ }^{[62]}$. Furthermore, a case series, done with the use of confirmed COVID-19 cases from the Bethesda Hospital Yogyakarta Indonesia, evaluated vitamin D status in COVID-19 patients. This study, which examined the medical records of 10 participants, reported a $90 \%$ prevalence rate of vitamin D deficiency from the observed cases ${ }^{[63]}$.

These findings suggest that both vitamins $C$ and D may have potential protective benefits against COVID-19 infections and may also serve to eliminate or reduce the occurrence of major complications that are observed with the disease.

\section{Discussion}

It is apparent that vitamins $\mathrm{C}$ and $\mathrm{D}$ both play beneficial roles in respiratory tract infections including those caused by the SARSCoV-2 virus. From these findings, there is no doubt that COVID19 patients may stand to significantly benefit from having normal levels of vitamins C and D before disease contraction or through the use of vitamins $\mathrm{C}$ and $\mathrm{D}$ supplementation following a diagnosis of COVID-19. Both vitamins support immune function, and their beneficial roles in COVID-19 may be derived from their effects on different immune cells and mediators.

Vitamin C exhibits antiviral effects as is demonstrated by studies in both animals and humans ${ }^{[1,64]}$. In addition, in patients suffering from COVID-19, this vitamin functions in decreasing immune responses that may promote pulmonary and systematic inflammation ${ }^{[65]}$. The blood of COVID-19 patients sometimes show high levels of inflammatory molecules. Evidence reveals that the treatment of COVID-19 patients with vitamin C, potentially decrease the levels of inflammatory molecules thus reducing inflammation ${ }^{[66]}$. Endocrine organs such as the pituitary and adrenal glands contain high levels of vitamin $\mathrm{C}$ and periods of physiological stress, including those caused by infections, result in the release of vitamin $\mathrm{C}$ from these glands therefore increasing its levels in the blood. The administration of glucocorticoid steroids has been proven effective in the treatment of COVID-19 infections. Vitamin $\mathrm{C}$ regulates the production of cortisol and as a result, promotes the anti-inflammatory and cellular protective effects of glucocorticoids ${ }^{[67]}$. Vitamin $C$ has anti-oxidant properties ${ }^{[68]}$ and can potentially prevent major complications of COVID-19. ARDS, which is a complication commonly observed with COVID-19 infections, is mainly caused by conditions that increase inflammation and oxidative stress ${ }^{[69]}$.

The literature shows that deficiencies in vitamin D are potentially linked to an increased risk for infections and autoimmune conditions ${ }^{[70]}$. COVID-19 patients may serve to benefit from the use of vitamin $\mathrm{D}$ as this vitamin functions to maintain cellular integrity and it also suppresses the cytokine storm thus supporting cellular immunity ${ }^{[59]}$. Furthermore, it induces Tregulatory cells and suppresses Th-1 cellular responses therefore, supporting adaptive immunity ${ }^{[71]}$. Vitamin D significantly impacts complications of COVID-19 such as ARDS and heart failure, as an association is already established between its deficiency and the presence of increased and persistent inflammation ${ }^{[72]}$. In addition to its effects on immune mediators, there are other mechanisms by which vitamin D provides potential benefits to COVID-19 patients. Similarly to SARS-CoV-1, SARS-CoV-2 utilizes angiotensin converting enzyme 2 (ACE-2) as a potential receptor for entering and infecting different cells throughout the body especially cells lining the respiratory and gastrointestinal tracts. When SARS-CoV2 binds ACE- 2 it causes a down regulation of ACE-2 thus promoting the development of pneumonia and lung injury ${ }^{[72]}$. Vitamin $\mathrm{D}$ in mice has been shown to decrease acute lung injury by disrupting the angiopoietin (Ang)-2-Tie-2 signalling pathway and through its negative effects on the renin angiotensin system (RAS) [73]. Furthermore, this vitamin potentially increases the levels of ACE2, MasR and Ang-(1-7) through its ability to enhance the activities of the ACE2/Ang-(1-7)/MasR axis, down regulates the ACE/Ang II/AT1R axis and inhibit renin. Therefore, through its influence on these various pathways, vitamin $\mathrm{D}$ decreases inflammation and may therefore be beneficial in the management of COVID-19 infections ${ }^{[72,74]}$.

Since an effective treatment and cure for the COVID-19 infection is yet to be established, prevention may be the only effective way for completely controlling this disease. Due to their established effects on the immune system and their proven effects on respiratory illnesses, there is no doubt that the use of vitamins D and $\mathrm{C}$ may prove beneficial in preventing the development of COVID-19 or may even help to reduce the severity of symptoms in COVID-19 patients. Several studies are currently being designed and executed as means of establishing the exact benefits of these vitamins in the treatment of SARS-CoV-2 infections. Results from these studies may serve to establish effective doses of these vitamins in addition to required routes of administration and safety levels that are required to effectively treat COVID-19 patients. Results may also be used in the preparations of guidelines for managing the disease which will determine if routine screening for serum levels of vitamins $\mathrm{C}$ and D should be carried out in COVID19 patients and whether or not these vitamins should be routinely supplemented in these patients. In the meantime, the incorporation of these vitamins as part of prevention or treatment regimens may serve great benefits to patients who are vulnerable or affected by the SARS-CoV-2 virus.

\section{Conclusion}

Evidence shows that vitamins $\mathrm{C}$ and $\mathrm{D}$ may provide some benefits to COVID-19 patients. Both vitamins have anti-viral effects and also possess the ability to regulate the immune system thus reducing inflammation. As a result, vitamins $\mathrm{C}$ and $\mathrm{D}$ reduce the occurrence and severity of COVID-19 complications, especially that of ARDS. Further studies will serve to prove the role of these vitamins in the management of SARS-CoV-2 infections and the doses of these vitamins that would be safe and effective; however, evidence from this review shows that these vitamins should be considered as part of the protocols for the prevention and management of COVID-19 infections.

\section{List of abbreviations}

ACE-2 - angiotensin converting enzyme 2

ARDS - acute respiratory distress syndrome

$\mathrm{FiO} 2$ - fraction of inspired oxygen

G-CSF - granulocyte colony-stimulating factor

HLA-DR allele - Human Leukocyte Antigen DR-isotype

IFN- $\gamma$ - interferon gamma

IL - Interleukin

IL-1 $\beta$ - interleukin 1 beta

RAS - renin angiotensin system

ROS - reactive oxygen species 
SARS-CoV-2 virus - Severe acute respiratory syndrome coronavirus 2

TNF- $\alpha$ - tumor necrosis factor $\alpha$

UVB - ultraviolet B

WHO - World Health Organization

\section{Conflict of Interest}

The authors of this paper declare no conflicts of interest.

\section{Funding}

No Source of Funding

\section{Authors' contributions}

Authors Merina Shrestha, Kaydeonne T. Ellis and Malcolm (Jr) Antoine carried out the systematic search of various data bases and collected the relevant data for composition of this manuscript. Data synthesis and manuscript writing was done by Chrystal AntoineFrank and Rimanatou Seyni Boureima. Chrystal Antoine-Frank assessed the final contents for accuracy and consistency. All authors read and agreed upon the final version of this manuscript.

\section{References}

[1] Gorton, H. C. \& Jarvis, K. The effectiveness of vitamin $\mathrm{C}$ in preventing and relieving the symptoms of virusinduced respiratory infections. J Manipulative Physiol Ther 22, 530-533 (1999).

[2] Velavan, T. P. \& Meyer, C. G. The COVID-19 epidemic. Trop Med Int Health 25, 278-280 (2020).

[3] WHO Coronavirus (COVID-19) Dashboard. https://covid19. who.int.

[4] Meyerowitz, E. A., Richterman, A., Gandhi, R. T. \& Sax, P. E. Transmission of SARS-CoV-2: A Review of Viral, Host, and Environmental Factors. Ann Intern Med (2020) doi:10.7326/M20-5008.

[5] Li, Q. et al. Early Transmission Dynamics in Wuhan, China, of Novel Coronavirus-Infected Pneumonia. N Engl J Med 382, 1199-1207 (2020).

[6] Guan, W. et al. Clinical characteristics of 2019 novel coronavirus infection in China. medRxiv 2020.02.06.20020974

(2020) doi:10.1101/2020.02.06.20020974.

[7] Chan, J. F.-W. et al. A familial cluster of pneumonia associated with the 2019 novel coronavirus indicating person-to-person transmission: a study of a family cluster. Lancet 395, 514-523 (2020).

[8] Chiscano-Camón, L., Ruiz-Rodriguez, J. C., RuizSanmartin, A., Roca, O. \& Ferrer, R. Vitamin C levels in patients with SARS-CoV-2-associated acute respiratory distress syndrome. Crit Care 24, 1-3 (2020).

[9] Martineau, A. R. \& Forouhi, N. G. Vitamin D for COVID-19: a case to answer? The Lancet Diabetes \& Endocrinology 8, 735-736 (2020).

[10] Boston, 677 Huntington Avenue \& Ma 02115 +1495-1000. Vitamin C. The Nutrition Source https://www.hsph.harvard.edu/nutritionsource/vitamin-c/ (2012).

[11] Cameron, E. \& Pauling, L. Ascorbic acid and the glycosaminoglycans. An orthomolecular approach to cancer and other diseases. Oncology 27, 181-192 (1973).
[12] Pauling, L. Vitamin C and common cold. JAMA 216, 332 (1971).

[13] Pauling, L. Diet, nutrition, and cancer. Am J Clin Nutr 30, 661-663 (1977).

[14] Pauling, L. Vitamin C therapy of advanced cancer. N Engl J Med 302, 694-695 (1980).

[15] Institute of Medicine (US) Panel on Dietary Antioxidants and Related Compounds. Dietary Reference Intakes for Vitamin C, Vitamin E, Selenium, and Carotenoids. (National Academies Press (US), 2000).

[16] Levine, M. New concepts in the biology and biochemistry of ascorbic acid. N Engl J Med 314, 892 902 (1986).

[17] Ascorbic acid and the catabolism of cholesterol. Nutr Rev 31, 154-156 (1973).

[18] Stevenson, N. R. \& Brush, M. K. Existence and characteristics of Na positive-dependent active transport of ascorbic acid in guinea pig. Am J Clin Nutr 22, 318 326 (1969).

[19] Malo, C. \& Wilson, J. X. Glucose modulates vitamin C transport in adult human small intestinal brush border membrane vesicles. J Nutr 130, 63-69 (2000).

[20] The Important Role of Vitamin D. Pharmacy Times https://www.pharmacytimes.com/publications/issue/2010 /february2010/otcfocusvitamind-0210.

[21] Bikle, D. Nonclassic Actions of Vitamin D. J Clin Endocrinol Metab 94, 26-34 (2009).

[22] Adams, J. S. \& Hewison, M. Update in Vitamin D. J Clin Endocrinol Metab 95, 471-478 (2010).

[23] Vitamins and minerals - Vitamin D. nhs.uk https://www.nhs.uk/conditions/vitamins-andminerals/vitamin-d/ (2017).

[24] Shakoor, H. et al. Immune-boosting role of vitamins D, C, E, zinc, selenium and omega-3 fatty acids: Could they help against COVID-19? Maturitas 143, 1-9 (2021).

[25] Huang, C. et al. Clinical features of patients infected with 2019 novel coronavirus in Wuhan, China. Lancet 395, 497-506 (2020).

[26] Rothan, H. A. \& Byrareddy, S. N. The epidemiology and pathogenesis of coronavirus disease (COVID-19) outbreak. J Autoimmun 109, 102433 (2020).

[27] Giamarellos-Bourboulis, E. J. et al. Complex Immune Dysregulation in COVID-19 Patients with Severe Respiratory Failure. Cell Host Microbe 27, 992-1000.e3 (2020).

[28] Washko, P., Rotrosen, D. \& Levine, M. Ascorbic acid transport and accumulation in human neutrophils. J Biol Chem 264, 18996-19002 (1989).

[29] Bergsten, P. et al. Millimolar concentrations of ascorbic acid in purified human mononuclear leukocytes. Depletion and reaccumulation. J Biol Chem 265, 2584 2587 (1990).

[30] Evans, R. M., Currie, L. \& Campbell, A. The distribution of ascorbic acid between various cellular components of blood, in normal individuals, and its relation to the plasma concentration. Br J Nutr 47, 473-482 (1982).

[31] Washko, P. W., Wang, Y. \& Levine, M. Ascorbic acid recycling in human neutrophils. J Biol Chem 268, 15531-15535 (1993).

[32] Leibovitz, B. \& Siegel, B. V. Ascorbic acid, neutrophil function, and the immune response. Int J Vitam Nutr Res 48, 159-164 (1978). 
[33] Carr, A. C., Shaw, G. M., Fowler, A. A. \& Natarajan, R. Ascorbate-dependent vasopressor synthesis: a rationale for vitamin $\mathrm{C}$ administration in severe sepsis and septic shock? Crit Care 19, 418 (2015).

[34] Carr, A. C. \& Maggini, S. Vitamin C and Immune Function. Nutrients 9, (2017).

[35] Bm, M. et al. Vitamin C: a novel regulator of neutrophil extracellular trap formation. Nutrients vol. 5 https://pubmed.ncbi.nlm.nih.gov/23939536/ (2013).

[36] $\mathrm{Mj}, \mathrm{H}$. et al. Technical advance: ascorbic acid induces development of double-positive $\mathrm{T}$ cells from human hematopoietic stem cells in the absence of stromal cells. Journal of leukocyte biology vol. 96 https://pubmed.ncbi.nlm.nih.gov/25157026/ (2014).

[37] M, T., N, M., E, G. \& I, Y. Enhancement by ascorbic acid 2-glucoside or repeated additions of ascorbate of mitogen-induced $\operatorname{IgM}$ and $\operatorname{IgG}$ productions by human peripheral blood lymphocytes. Japanese journal of pharmacology vol.

66 https://pubmed.ncbi.nlm.nih.gov/7723222/ (1994).

[38] Jd, C., M, C., B, B. \& At, V. Ascorbic acid is a potent inhibitor of various forms of $\mathrm{T}$ cell apoptosis. Cellular immunology vol. 194 https://pubmed.ncbi.nlm.nih.gov/10357874/ (1999).

[39] Animashaun, A., Kelleher, J., Heatley, R. V., Trejdosiewicz, L. K. \& Losowsky, M. S. The effect of zinc and vitamin $\mathrm{C}$ supplementation on the immune status of patients with Crohn's disease. Clin Nutr 9, 137146 (1990).

[40] N, M., Ac, M., Ap, B. \& R, O. Comparative effect of fucoxanthin and vitamin $\mathrm{C}$ on oxidative and functional parameters of human lymphocytes. International immunopharmacology vol. 22 https://pubmed.ncbi.nlm.nih.gov/24975831/ (2014).

[41] Chen, S. et al. Modulatory effects of 1,25dihydroxyvitamin $\mathrm{D}_{3}$ on human $\mathrm{B}$ cell differentiation. $\mathrm{J}$ Immunol 179, 1634-1647 (2007).

[42] Rigby, W. F., Stacy, T. \& Fanger, M. W. Inhibition of T lymphocyte mitogenesis by 1,25-dihydroxyvitamin $\mathrm{D}_{3}$ (calcitriol). J Clin Invest 74, 1451-1455 (1984).

[43] Boonstra, A. et al. 1alpha,25-Dihydroxyvitamin d3 has a direct effect on naive CD4(+) $\mathrm{T}$ cells to enhance the development of Th2 cells. J Immunol 167, 4974-4980 (2001).

[44] Aranow, C. Vitamin D and the Immune System. J Investig Med 59, 881-886 (2011).

[45] Murni, I. K., Prawirohartono, E. P. \& Triasih, R. Potential Role of Vitamins and Zinc on Acute Respiratory Infections Including Covid-19. Global Pediatric Health 8, 2333794X211021739 (2021).

[46] Sasazuki, S. et al. Effect of vitamin C on common cold: randomized controlled trial. European Journal of Clinical Nutrition 60, 9-17 (2006).

[47] Vorilhon, P. et al. Efficacy of vitamin C for the prevention and treatment of upper respiratory tract infection. A meta-analysis in children. Eur J Clin Pharmacol 75, 303-311 (2019).

[48] Hemilä, H. Vitamin C supplementation and respiratory infections: a systematic review. Mil Med 169, 920-925 (2004).

[49] Ran, L. et al. Extra Dose of Vitamin C Based on a Daily Supplementation Shortens the Common Cold: A Meta-
Analysis of 9 Randomized Controlled Trials. Biomed Res Int 2018, (2018).

[50] Laaksi, I. et al. An association of serum vitamin D concentrations < $40 \mathrm{nmol} / \mathrm{L}$ with acute respiratory tract infection in young Finnish men. Am J Clin Nutr 86, 714 717 (2007).

[51] Urashima, M. et al. Randomized trial of vitamin D supplementation to prevent seasonal influenza $A$ in schoolchildren. Am J Clin Nutr 91, 1255-1260 (2010).

[52] Zhou, J. et al. Preventive Effects of Vitamin D on Seasonal Influenza A in Infants: A Multicenter, Randomized, Open, Controlled Clinical Trial. Pediatr Infect Dis J 37, 749-754 (2018).

[53] Arihiro, S. et al. Randomized Trial of Vitamin D Supplementation to Prevent Seasonal Influenza and Upper Respiratory Infection in Patients With Inflammatory Bowel Disease. Inflamm Bowel Dis 25, 1088-1095 (2019).

[54] Loeb, M. et al. Effect of Vitamin D supplementation to reduce respiratory infections in children and adolescents in Vietnam: A randomized controlled trial. Influenza Other Respir Viruses 13, 176-183 (2019).

[55] High-dose vitamin $C$ infusion for the treatment of critically ill COVID-19. (2020) doi:10.21203/rs.3.rs52778/v1.

[56] Khan, H. M. W., Parikh, N., Megala, S. M. \& Predeteanu, G. S. Unusual Early Recovery of a Critical COVID-19 Patient After Administration of Intravenous Vitamin C. Am J Case Rep 21, e925521-1-e925521-6 (2020).

[57] Hiedra, R. et al. The use of IV vitamin C for patients with COVID-19: a case series. Expert Rev Anti Infect Ther 18, 1259-1261 (2020).

[58] Hemilä, H. \& Chalker, E. Vitamin C may reduce the duration of mechanical ventilation in critically ill patients: a meta-regression analysis. Journal of Intensive Care 8, 15 (2020).

[59] Grant, W. B. et al. Evidence that Vitamin D Supplementation Could Reduce Risk of Influenza and COVID-19 Infections and Deaths. Nutrients 12, (2020).

[60] Meltzer, D. O. et al. Association of Vitamin D Status and Other Clinical Characteristics With COVID-19 Test Results. JAMA Netw Open 3, e2019722 (2020).

[61] Carpagnano, G. E. et al. Vitamin D deficiency as a predictor of poor prognosis in patients with acute respiratory failure due to COVID-19. J Endocrinol Invest 1-7 (2020) doi:10.1007/s40618-020-01370-x.

[62] Entrenas Castillo, M. et al. "Effect of calcifediol treatment and best available therapy versus best available therapy on intensive care unit admission and mortality among patients hospitalized for COVID-19: A pilot randomized clinical study". J Steroid Biochem Mol Biol 203, 105751 (2020).

[63] Pinzon, R. T., Angela \& Pradana, A. W. Vitamin D deficiency among patients with COVID-19: case series and recent literature review. Tropical Medicine and Health 48, 102 (2020).

[64] Kim, Y. et al. Vitamin C Is an Essential Factor on the Anti-viral Immune Responses through the Production of Interferon- $\alpha / \beta$ at the Initial Stage of Influenza A Virus (H3N2) Infection. Immune Netw 13, 70-74 (2013). 
[65] García, L. F. Immune Response, Inflammation, and the Clinical Spectrum of COVID-19. Front Immunol 11, (2020).

[66] Hiedra, R. et al. The use of IV vitamin $\mathrm{C}$ for patients with COVID-19: a case series. Expert Rev Anti Infect Ther 1-3 doi:10.1080/14787210.2020.1794819.

[67] Vitamin C and COVID-19: A Review. News-Medical.net https://www.news-medical.net/news/20201023/VitaminC-and-COVID-19-A-Review.aspx (2020).

[68] Colunga Biancatelli, R. M. L., Berrill, M. \& Marik, P. E. The antiviral properties of vitamin C. Expert Rev Anti Infect Ther 18, 99-101 (2020).

[69] Calder, P. C. Nutrition, immunity and COVID-19. BMJ Nutrition, Prevention \& Health 3, (2020).

[70] Baeke, F., Takiishi, T., Korf, H., Gysemans, C. \& Mathieu, C. Vitamin D: modulator of the immune system. Curr Opin Pharmacol 10, 482-496 (2010).
[71] Cantorna, M. T., Snyder, L., Lin, Y.-D. \& Yang, L. Vitamin $\mathrm{D}$ and $1,25(\mathrm{OH}) 2 \mathrm{D}$ regulation of $\mathrm{T}$ cells. Nutrients 7, 3011-3021 (2015).

[72] Malek Mahdavi, A. A brief review of interplay between vitamin $\mathrm{D}$ and angiotensin-converting enzyme 2: Implications for a potential treatment for COVID-19. Rev Med Virol (2020) doi:10.1002/rmv.2119.

[73] Kong, J. et al. VDR attenuates acute lung injury by blocking Ang-2-Tie-2 pathway and renin-angiotensin system. Mol Endocrinol 27, 2116-2125 (2013).

[74] Iddir, M. et al. Strengthening the Immune System and Reducing Inflammation and Oxidative Stress through Diet and Nutrition: Considerations during the COVID-19 Crisis. Nutrients 12, (2020). 